\title{
Nuclear Medicine Methods for Assessment of Chronic Chagas Heart Disease
}

\author{
Marcus Vinicius Simões, 몬 Denise Mayumi Tanaka, José Antonio Marin-Neto \\ Universidade de São Paulo, São Paulo, SP - Brazil.
}

\section{Abstract}

Several different imaging methods can be used to evaluate patients with Chagas heart disease (CHD) for diagnostic and prognostic purposes, including plain chest radiography; echocardiography; myocardial perfusion scintigraphy, for detection of ischemia and fibrosis; radionuclide gated-angiography, for evaluation of biventricular function; ${ }^{123}$ I-MIBG labeling of sympathetic myocardial innervation; MRI, for detection and quantitation of myocardial fibrosis; and coronary angiography. This study aims to review the contributions of these nuclear medicine methods to understanding of the pathophysiology of chronic Chagas cardiomyopathy (CCC). Careful analysis and integration of findings provided by these imaging methods in patients with CCC at different stages has contributed significantly to improving understanding of several peculiarities of the disease. Clinical and experimental studies in animal models show that perfusion abnormalities detected in association with dysfunctional but viable myocardium are a common finding in CCC patients and correspond to areas of cardiac sympathetic denervation, as assessed by ${ }^{123}$ I-MIBG imaging. Furthermore, recent reports have demonstrated a close relationship between coronary microvascular disturbances and myocardial inflammation. Thus, ongoing research, mainly focused on refinements of ${ }^{18} \mathrm{~F}$-FDF -PET techniques and further exploration of nuclear methods, such as SPECT, have the potential to contribute to detection and monitoring of early subclinical myocardial damage thereby

\section{Keywords}

Chagas Cardiomyopathy; Chagas Disease; Diagnostic, Imaging; Prognosis; Echocardiography; Myocardial Perfusion. enabling evaluation of therapeutic strategies targeting inflammation and microvascular ischemia that could result in better prognostic stratification of patients with CHD.

\section{Introduction}

Chagas disease (CD) is caused by a protozoan parasite, Trypanosoma cruzi (T. cruzi), which is mainly transmitted among human beings through a triatomine vector, but other transmission routes also exist, including congenital, blood transfusion, oral transmission, laboratory contamination, and organ transplantation. ${ }^{1}$ The disease is a serious health problem that is still endemic in many regions of Latin America, where some 8 to 10 million people are estimated to be infected, and it represents an emerging public health issue in nonendemic countries such as the United States and European and Asian countries ${ }^{2,3}$ because of globalization and migratory waves from endemic regions.

CD presents two distinct phases. The acute phase is of short duration, lasting from 1 to 3 months, and is usually a benign febrile disease, but can rarely involve serious cardiovascular and neurologic complications caused by intense inflammatory changes secondary to $T$. cruzi parasitism in multiple organs and systems. In most cases, the disease will be asymptomatic in the chronic phase, without clinical evidence of structural organ damage throughout the individual's life, constituting the indeterminate form of Chagas disease (IFCD). ${ }^{4}$ However, approximately $30 \%$ of chronically infected patients develop symptoms, with progressive cardiac and/or digestive organ complications. The cardiac form is the most relevant clinical manifestation of the disease in the chronic phase and may manifest as dilated cardiomyopathy arising about 2 to 3 decades after the initial infection. ${ }^{5}$ 
The mechanisms of injury that lead to such a delayed development of myocardial damage are an intriguing aspect of the pathogenesis of CCC. The main pathologic feature of CCC is a low intensity and incessant chronic focal myocarditis that is initially silent but causes progressive myocardial damage with extensive reparative and reactive interstitial fibrosis that ultimately leads to a severe form of dilated cardiomyopathy. ${ }^{6}$ Impairment of left ventricular wall motion that predominates in the inferior, posterior-lateral, and apical regions is a common finding in the early stages of CCC, preceding global cardiac systolic dysfunction. Additionally, the finding of an isolated, thin walled, left ventricular apical aneurysm, with a "finger glove" appearance, is considered a hallmark of the disease. ${ }^{7}$

The earliest clinical manifestations of CCC include cardiac conduction disorders, ${ }^{8} \mathrm{LV}$ wall motion abnormalities, ${ }^{9}$ and diastolic dysfunction. ${ }^{10}$ Sudden death due to complex arrhythmias may occur during the entire clinical course. Late manifestations include an important dilatation of cardiac chambers, as well as changes in their function, resembling dilated cardiomyopathy in clinical terms. The most common clinical manifestations in this phase are signs and symptoms of heart failure, systemic thromboembolism episodes, atrioventricular blocks, and severe ventricular arrhythmias. ${ }^{11,12}$

Several imaging methods can be used to evaluate patients with Chagas heart disease for diagnostic and prognostic purposes. ${ }^{13}$ Radionuclide imaging is an important tool employed to investigate and characterize several aspects of cardiac involvement in CCC patients. This primarily includes radionuclide ventriculography for bi-ventricular function evaluation, myocardial perfusion scintigraphy for recognition of coronary microvascular disturbances, ${ }^{123}$ I-MIBG for imaging of cardiac sympathetic innervation, and, most recently, use of PET imaging has been suggested as a promising tool for detecting myocardial inflammation.

\section{Radionuclide Ventriculography}

Planar radionuclide ventriculography was one of the first nuclear imaging techniques employed to explore right and left ventricular function and to assess wallmotion abnormalities in patients with CCC. ${ }^{14}$ That study ${ }^{14}$ reported that radionuclide ventriculography provided reliable information on global LV function and regional wall motion abnormalities, with good correlation between radiologic contrast ventriculography performed during cardiac catheterization and radionuclide ventriculography results.

A prominent feature in patients with Chagas disease is right ventricular dysfunction that may occur in the absence of any detectable LV disorder. ${ }^{15}$ These findings were described by Marin Neto et $\mathrm{al}^{15,16}$ and confirmed previous studies employing planar rest gated equilibrium radionuclide angiography with ${ }^{99 m}$ Technetium. ${ }^{15,16}$ Both studies showed that reduced right ventricular ejection fraction can be the only functional cardiac disorder detectable in some chronic Chagas patients with the indeterminate or the digestive forms, without any other clinical signs of heart disease.

Application of gated SPECT technique enables tomographic acquisition of blood pool imaging and more detailed assessment of LV dysfunction. Previous studies have used this approach to achieve a more accurate correlation between SPECT myocardial perfusion changes and regional wall motion abnormalities in cohorts of CCC patients with varying myocardial disease severity, demonstrating a close topographic relationship between regional wall motion impairment and reversible or fixed myocardial perfusion defects. ${ }^{17}$

More recently, radionuclide ventriculography image acquisition with the SPECT technique, which produces tomographic images of the ventricular cavity, co-registered with myocardial perfusion gated-SPECT imaging of the ventricular walls, were used to provide complementary information allowing characterization of left ventricle apical aneurysm, ${ }^{17}$ the most notable segmental disorder in chronic Chagas' heart disease. That study described severe perfusion defects in the aneurysmal apical region, although relatively smaller when compared to the aneurysm size, identified by radionuclide ventriculography. Notably, the myocardial segments surrounding the aneurysm have preserved segmental wall motion, resulting in an aneurysm with a narrow neck, and the dyskinetic ventricular cavity has a "glove finger" appearance (figure 1). This aspect clearly diverges from what is classically found in apical aneurysms secondary to ischemic heart disease, in which the LV wall segments of the aneurysmal formation show typically dyskinetic movement. It is important to note that early detection of the aneurysm using several diagnostic nuclear imaging methods is clinically relevant because the apical region is somewhat more difficult to assess when standard echocardiographic methods are used. Furthermore, detection enables risk stratification with therapeutic implications, mainly with relation to 


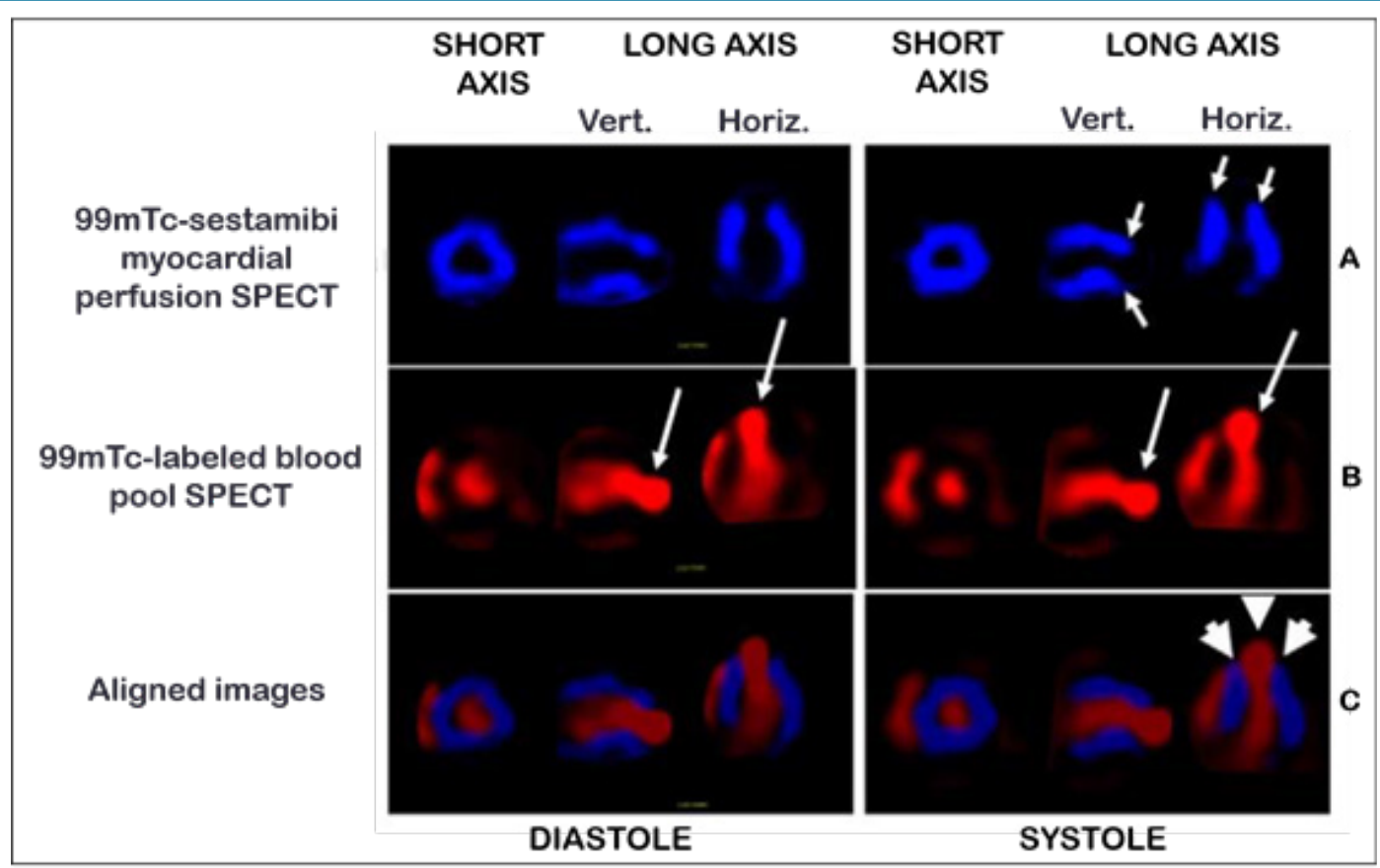

Figure 1 - Tomographic images. The left panels show the diastolic frames, and right panels are the systolic frames. A: Myocardial perfusion SPECT showing a severe perfusion defect involving a moderate portion of the cardiac apex, with preserved motion of the segments surrounding it (short arrows). B: Radionuclide ventriculography revealing an apical aneurysm extending beyond the left ventricular walls (long arrows). C: Co-registered images showing topographic correlation between the aneurysm cavity, the myocardial segments that form its "neck", and the perfusion defects (wide arrows). Adapted with permission from Simoes, et al. ${ }^{17}$

the risk of thromboembolism and potential indication of anticoagulation therapy. ${ }^{17}$

\section{Myocardial Perfusion Scintigraphy}

One particular feature of CCC is the clinical and laboratorial presentation masquerading as coronary artery disease. ${ }^{18}$ Previous reports called attention to the occurrence of regional LV dyssynergia associated with rest (fixed) myocardial perfusion defects, mainly in the apical and basal portion of the posterior-lateral wall, that could be misinterpreted as regions of scar tissue resulting from previous myocardial infarction and atherosclerotic coronary artery disease. ${ }^{19}$ Furthermore, precordial chest pain is a common manifestation and mimics acute or chronic coronary syndromes usually associated with classic coronary artery disease, but with normal epicardial coronary arteries on angiography. ${ }^{20,21}$ In this scenario, several clinical studies using myocardial perfusion scintigraphy reported findings of reversible defects compatible with reversible myocardial ischemia in CCC patients exhibiting normal coronary arteries, indicating the presence of coronary microvascular dysfunction.
There is now sound evidence in the literature suggesting that myocardial perfusion disturbances (MPD) caused by microvascular dysfunction participate in the pathogenesis of the myocardial damage process that ultimately leads to CCC. ${ }^{6,22}$ This hypothesis is also supported by pioneering autopsy studies showing a topographic correlation between coronary microvascular obstruction and ischemic myocardial lesions in CCC patients, ${ }^{23,}{ }^{24}$ Use of scintigraphy myocardial perfusion imaging makes a substantial contribution towards confirming the participation of microvascular disturbances in the pathogenesis of CCC.

\section{Clinical Studies}

In clinical settings, the first report of the occurrence of MPD in CCC patients employed assessment of global myocardial flow using ${ }^{86}$ Rubidium and was published by Kuschnir and colleagues. ${ }^{25}$ They observed a reduction in myocardial perfusion at rest and during physical exercise in comparison with healthy individuals. After that, remarkable myocardial perfusion abnormalities in CCC patients with angiographically normal coronary arteries were reported in $30-50 \%$ of patients by several 
independent investigations and the presence of perfusion disturbances due to coronary microvascular dysfunction was postulated. ${ }^{21,26}$

Marin-Neto et al. studied 23 patients with CCC and normal coronary arteries using thallium-201 stressredistribution planar images and observed MPD in all cases. ${ }^{26}$ Fixed defects, predicting regional myocardial fibrosis, were mainly found in myocardial regions exhibiting akinesis or dyskinesis, while reversible myocardial ischemia located in LV segments with less severe wall motion impairment was detected in 8 (35\%) patients. Corroborating those findings, a further study with thallium-201SPECT investigated 37 patients with various stages of CCC, including 12 patients without any apparent cardiac involvement, 13 patients with regional LV dysfunction, but normal global LV systolic function, and 13 patients with advanced CCC with reduced LVEF. ${ }^{19}$ All types of perfusion defects (fixed, paradoxical, and reversible) were again observed in $78 \%$ of the patients. Additionally, a significant topographic correlation was observed between perfusion disturbances and wall motion abnormalities in the apical and inferiorposterior-lateral LV segments. Notably, reversible MPD were detected in $42 \%$ of $12 \mathrm{CHD}$ patients who otherwise had no evidence of myocardial disease (Figure 2). These reversible ischemic defects were mostly observed in the apical and inferior-posterior LV segments that correspond to regions in which regional contractile dysfunction is more frequently found in later stages of CCC, ${ }^{26}$ strongly suggesting that myocardial perfusion disturbance precedes development of regional myocardial damage in CCC. This concept was further supported by another investigation using ${ }^{99 \mathrm{~m} T c}$-sestamibi myocardial perfusion SPECT in patients with the indeterminate form and myocardial reversible perfusion defects affecting LV segments also exhibiting wall motion abnormalities were reported in $25 \%$ of patients. ${ }^{27}$ Overall, these findings support the concept that MPD occurs in early stages of CCC and may precede regional LV dysfunction. ${ }^{22}$

Hiss et al. ${ }^{28}$ provided additional evidence supporting this hypothesis with a longitudinal, retrospective study of 36 patients with CHD who were initially evaluated with stress-rest myocardial perfusion scintigraphy and then had the nuclear scans repeated after a mean period of 5.6 years. ${ }^{28}$ At baseline, 20 of the 36 patients (56\%) exhibited reversible MPD involving an average of $10.2 \%$ of the LV area, as determined by the SPECT technique. Over the course of the follow-up period, the LVEF declined significantly from 55\% $\pm 11 \%$ to $50 \% \pm$ $13 \%$ and several of the initially reversible defects became fixed over time. Moreover, there was a correlation between the increase in the perfusion defect area at rest and the reduction in LV ejection fraction. Most notably, presence of ischemia in the initial evaluation exhibited a

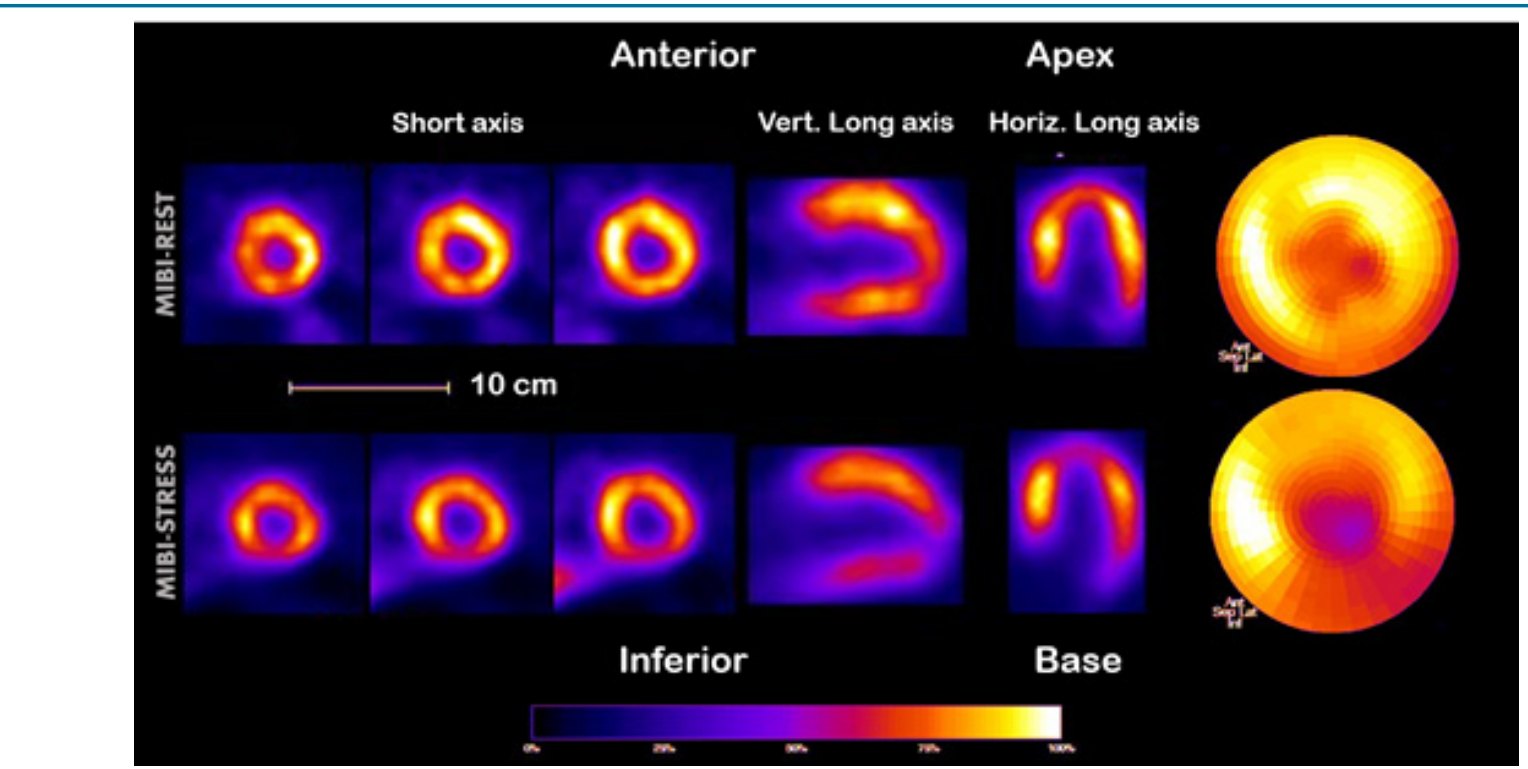

Figure 2 - Representative slices of stress-rest SPECT imaging with 99mTc-sestamibi of a 66-year-old female patient with CCC and normal coronary arteries on angiography, presenting reversible perfusion defects in the segments of the apex and inferior wall, involving $22 \%$ of the LV polar map surface area (stress). 
topographic association with later development of wall motion abnormality in the same area; of the 47 segments presenting reversible perfusion defects in the initial study, 32 (68\%) progressed to perfusion defects at rest, and of the 469 segments not showing reversibility in the initial study, only 41 (8.7\%) had the same progression. In summary, in this longitudinal study, deterioration of LV systolic function over time was associated with both the presence of reversible ischemic defects at the initial assessment and with an increase in the extent of rest perfusion defects indicative of regional myocardial fibrosis during follow-up.

There is therefore compelling evidence indicating that coronary microvascular disturbances play a significant role in myocardial damage and progression of CCC, and that SPECT myocardial perfusion imaging is a promising tool for early detection, risk stratification, and monitoring of the progression of CCC. ${ }^{29}$

\section{Experimental Studies}

More recently, experimental models of chronic Chagas heart disease in small animals were investigated in vivo employing high-resolution scintigraphy images, enabling histological correlation of the perfusion disturbance and providing relevant information to clarify the participation of perfusion and inflammation derangements in the pathophysiological mechanism of CCC.

A pioneering study by Lemos de Oliveira et al., ${ }^{30}$ reported detection of rest MPD using in vivo highresolution ${ }^{99 m}$ Tc-sestamibi SPECT myocardial perfusion imaging in an experimental model of CCC in hamsters. In that cross-sectional study, Syrian hamsters were investigated in the chronic phase at time windows of 6 and 10 months after experimental infection with $T$. cruzi. The results showed severe rest MPD occurring at similar rates as have previously been reported in humans at comparable stages of development of CCC $(50 \%)$, and involving segments of the anterior-lateral and apical walls. The results of the in vivo images were topographically correlated with data from quantitative histopathological analysis, revealing no areas of transmural fibrosis in those segments with MPD, but showing higher intensity of inflammatory infiltrate. ${ }^{30}$

To understand those findings in greater depth, Tanaka et al., ${ }^{31}$ tested the effects of prolonged use of dipyridamole, a coronary microvascular dilating drug, on the rest MPD of CCC hamsters. Six months after experimental T. cruzi infection, infected animals (assigned to receive either dipyridamole or placebo) had larger areas of perfusion defect than the non-infected control animal groups and preserved LV systolic function. After treatment, the infected animals that received dipyridamole exhibited a significant reduction of the MPD area (from 17.3 \pm 3.7 to $6.8 \pm 2.1 \%, p=0.001$ ) (Figure 3)..$^{31}$

In summary, these findings support the hypothesis that areas with rest MPD correspond to viable but hypoperfused myocardium, similar to the hibernating myocardial phenomenon found in ischemic disease, and that coronary microvascular disturbance is closely linked to inflammation, playing a role in the pathophysiology of myocardial damage in CCC. Future research should target elucidation of the causal relationship between inflammation and microvascular ischemia and seek to discover the contribution made by each disorder to the pathogenesis of CCC.

Myocardial Sympathetic Innervation Imaging with ${ }^{123}$ I-MIBG

Cardiac autonomic denervation is another remarkable feature of CCC and was first described in human autopsy studies. ${ }^{32}$ Although this finding is not specific for CCC, the intensity of the cardiac neuronal depopulation surpasses that seen in any other etiology of heart disease. ${ }^{33,34}$ Striking depopulation of the parasympathetic neuronal bodies in the atrial tissue and of the sympathetic paravertebral ganglia has been reported by several independent investigators. As a consequence of these anatomical findings, several methods of investigation have been employed in previous studies and showed functional abnormalities of reflex autonomic control of the heart rate. ${ }^{6}$ Myocardial scintigraphy with ${ }^{123} \mathrm{I}-\mathrm{MIBG}$ (MIBG) has been used in several stages of CCC to noninvasively assess myocardial sympathetic innervation and provide accurate information about the integrity of sympathetic nerve fibers and the degree of activation of cardiac sympathetic innervation. ${ }^{19,35}$

Assessment of cardiac sympathetic innervation in patients with CCC using ${ }^{123}$ I-MIBG scintigraphy was first described by Simões et al. ${ }^{12}$ In that study, a series of 37 patients with several degrees of LV dysfunction underwent planar and SPECT ${ }^{123}$ I-MIBG imaging and the results were correlated with myocardial perfusion scintigraphy and LV function assessments. It was observed that defects of MIBG uptake were seen in the majority of patients: in 33\% of the patients without any other evidence of cardiac disease and $77 \%$ of the patients 

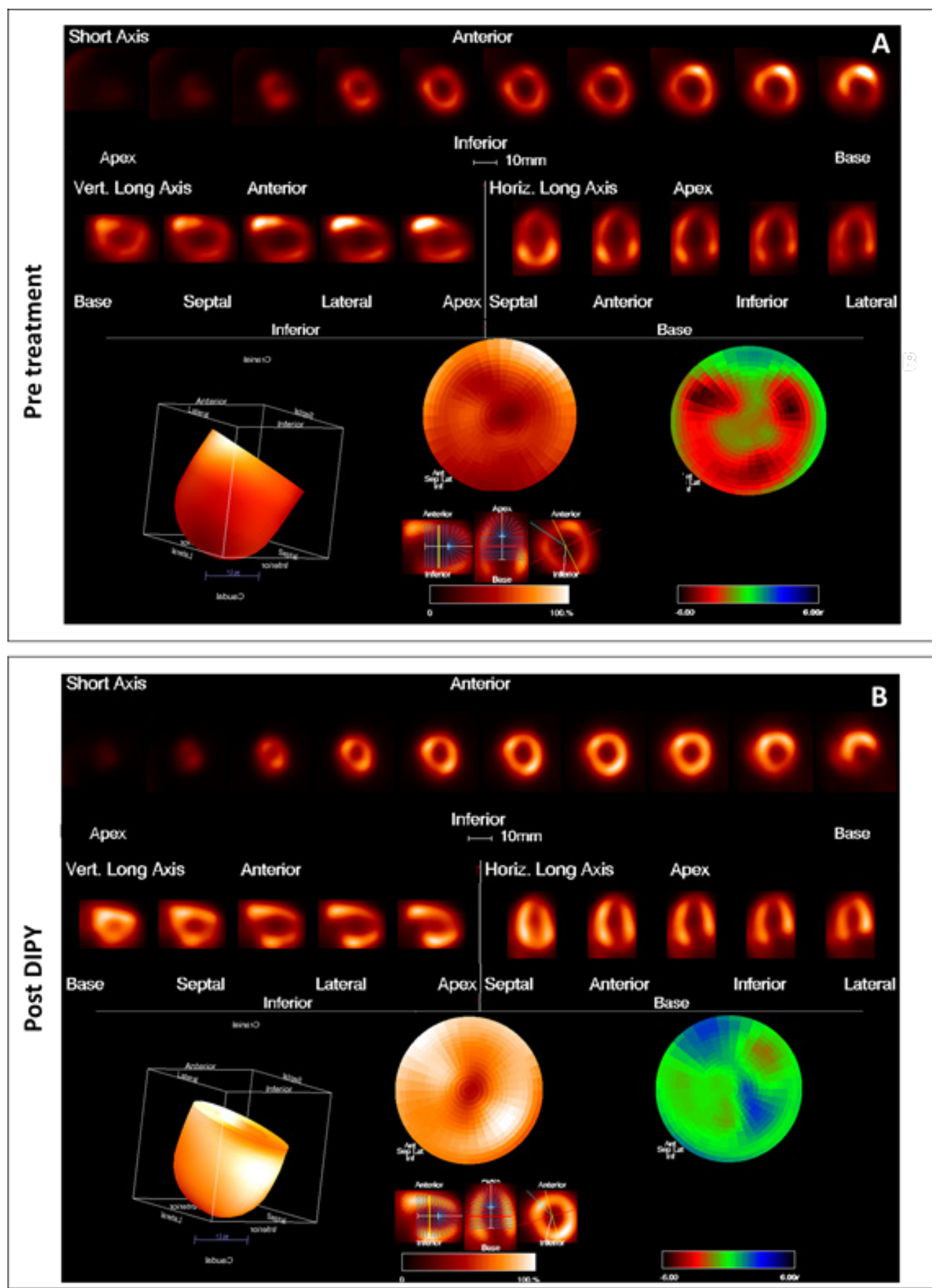

Figure 3 - Illustrative images from a myocardial perfusion study of a T. cruzi infected animal at 6 months post infection at (A) pre-treatment and (B) after 4 weeks of dipyridamole treatment. Representative slices of tomographic images (SPECT) are shown. The images were obtained along short axis and on vertical and horizontal long axes, with the resulting polar map shown in the lower panel of the figure. The dipyridamole-treated animal presented a severe perfusion defect involving the septal, anterior, lateral, and apical wall at baseline evaluation, with a striking reduction of perfusion impairment in the images acquired in the post-treatment evaluation. 
with regional LV wall motion disturbance. Furthermore, patients with more severe LV dysfunction had a higher prevalence of MIBG defects (92\%). Notably, there was a topographic correlation between areas of myocardial sympathetic denervation and areas exhibiting fixed and reversible myocardial perfusion scintigraphy defects and abnormal segmental LV wall motion predominantly involving the inferior, posterior-lateral, and apical LV walls. These initial results indicated that sympathetic denervation is an early derangement in the pathophysiology of CCC, before development of regional LV contraction disturbance or global dysfunction (Figure 4). ${ }^{19}$ This hypothesis was corroborated by the results of an independent study showing abnormal MIBG uptake in most CCC patients with no other signs of cardiac involvement. ${ }^{36}$

A more recent study employed image co-registration in 13 patients with CCC to investigate quantitative and topographic correlations between areas of cardiac sympathetic denervation using ${ }^{123} \mathrm{I}-\mathrm{MIBG}-\mathrm{SPECT}$, myocardial hypoperfusion using ${ }^{99 \mathrm{~m}} \mathrm{Tc}$-sestamibi-SPECT, and myocardial scarring using magnetic resonance imaging (MRI) ${ }^{37}$ The results showed strong topographic agreement between areas of denervation and areas of stress-hypoperfused myocardium, corresponding to $60.8 \%$ of the denervated area. MRI showed that only $16.1 \%$ of the denervated area corresponded to areas of fibrosis. $^{37}$
Miranda et al., ${ }^{35}$ addressed the correlation between presence and extent of myocardial sympathetic denervation and occurrence of severe ventricular arrhythmia in CCC patients with normal or mildly reduced LVEF. ${ }^{35}$ In this study, patients with sustained ventricular tachycardia had higher ${ }^{123} \mathrm{I}-\mathrm{MIBG}$ summed defect scores than 11 patients without sustained ventricular tachycardia. These findings indicated a relevant role of myocardial sympathetic denervation as a trigger mechanism for malignant ventricular arrhythmia in CCC. ${ }^{35}$

Another recent study was conducted with the objective of investigating the correlation between the extent of cardiac sympathetic denervation and occurrence of ventricular arrhythmia of varying severity in CCC patients. In that study, 15 CCC patients with sustained ventricular tachycardia had larger areas of viable but denervated myocardium, assessed by the summed difference defect scores between MIBG and ${ }^{99 m}$ Tc-sestamibi SPECT images (20.0 \pm 8.0), than 17 CCC patients with a less severe form of ventricular arrhythmia, i.e., non-sustained ventricular tachycardia, (11.0 $\pm 8.0, \mathrm{p}<0.05)$, and also had larger areas than CCC patients without any repetitive ventricular arrhythmia, according to Holter monitoring $(2.0 \pm 5.0, \mathrm{p}<0.0001) .{ }^{38}$ It is important to emphasize that severe ventricular arrythmia can lead to sudden death in early phases of the disease, even in patients with preserved global left ventricular function, ${ }^{39}$ and so a risk stratifying tool such as that provided by assessment

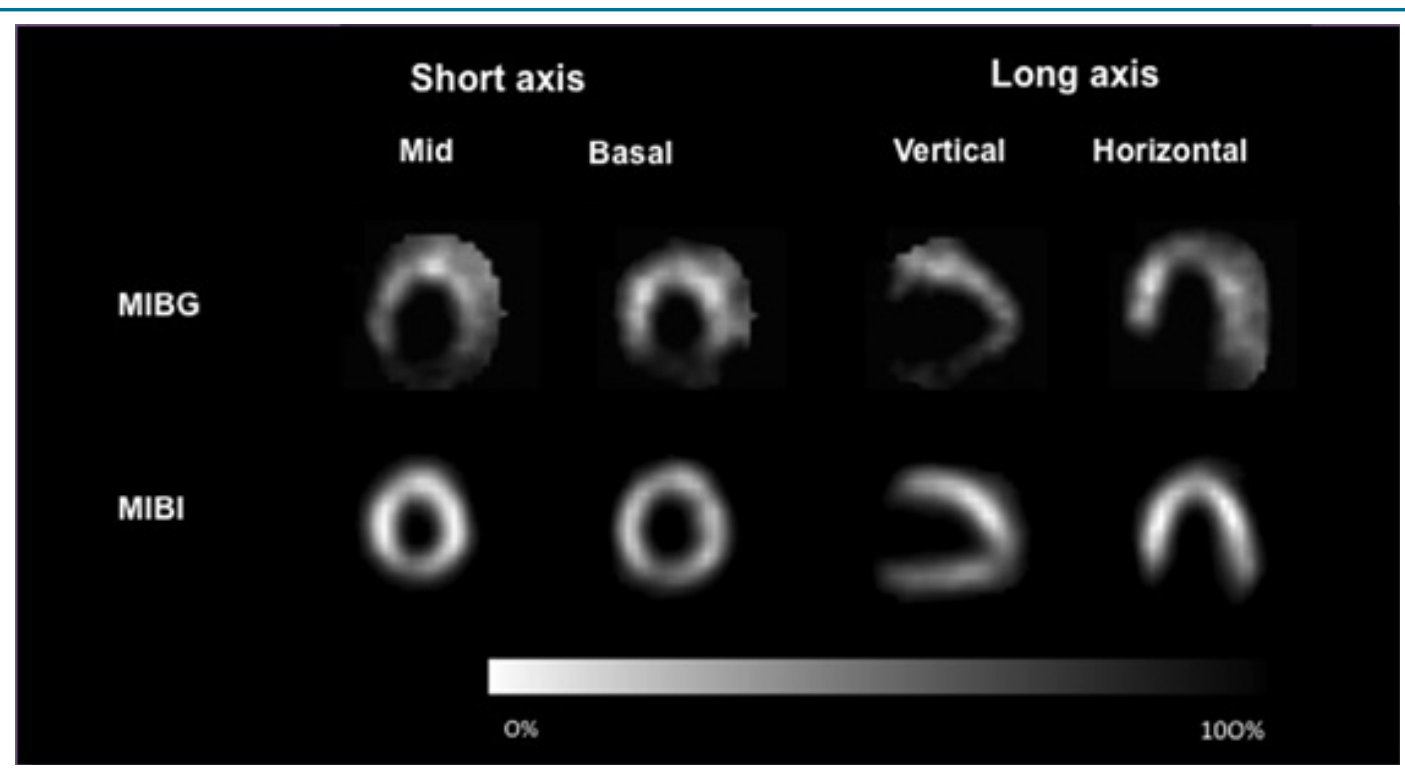

Figure 4 - Representative image of ${ }^{123}$ I-MIBG (upper panel) and ${ }^{99 m}$ Tc-sestamibi SPECT (lower panel) showing normal Tc99mSestamibi uptake and severe ${ }^{123}$ I-MIBG uptake defects in the segments of the inferior, inferior-lateral, and septal inferior walls, indicating the presence of viable but denervated myocardium. 
of cardiac sympathetic denervation could be useful in this clinical scenario.

In summary, the available data reinforces the notion that the extent of myocardial sympathetic denervation is closely correlated with the incidence of malignant ventricular arrhythmia in CCC patients, and MIBG scintigraphy may be a potential tool for stratification of arrhythmic sudden cardiac death risk.

\section{Imaging Myocardial Viability with FDG-PET}

Positron emission tomography (PET) is a useful tool for in vivo investigation of myocardial metabolism. ${ }^{40}$ The metabolic state of the myocardium can be evaluated by regional uptake of ${ }^{18} \mathrm{~F}-\mathrm{FDG}$, which is an analogue of glucose. ${ }^{41}$ Thus, regions with preserved uptake of ${ }^{18}$ F-FDG correspond to metabolically viable myocardium.

In experimental studies, a variety of strategies can be employed to improve ${ }^{18}$ F-FDG accumulation by cardiomyocytes, such as use of different anesthetic agents, such as isoflurane, enhancing detection of myocardial metabolic viability. ${ }^{42}$ In this scenario, Lemos de Oliveira, et al. ${ }^{30}$ studied a model of chronic Chagas cardiomyopathy in hamsters using in vivo imaging methods including resting ${ }^{99 \mathrm{~m}} \mathrm{Tc}$-sestamibi and ${ }^{18} \mathrm{~F}$-FDG PET under isoflurane anesthesia, observing preserved or only mild reduction of ${ }^{18}$ F-FDG uptake in regions with severe myocardial prefusion defects at rest, showing the presence of myocardial viability in those regions (Figure 5). Moreover, histopathological analysis of fibrosis reinforced these findings, since no coalescent fibrosis was found. ${ }^{30}$

\section{Imaging Cardiac Inflammation with FDG-PET}

To further improve understanding of the relationship between inflammation and progression of CCC in humans, it is essential to employ an imaging method able to detect and quantify myocardial inflammation in vivo. In this scenario, several studies have demonstrated the possibility of imaging myocardial inflammation using ${ }^{18} \mathrm{~F}-\mathrm{FDG}$-PET

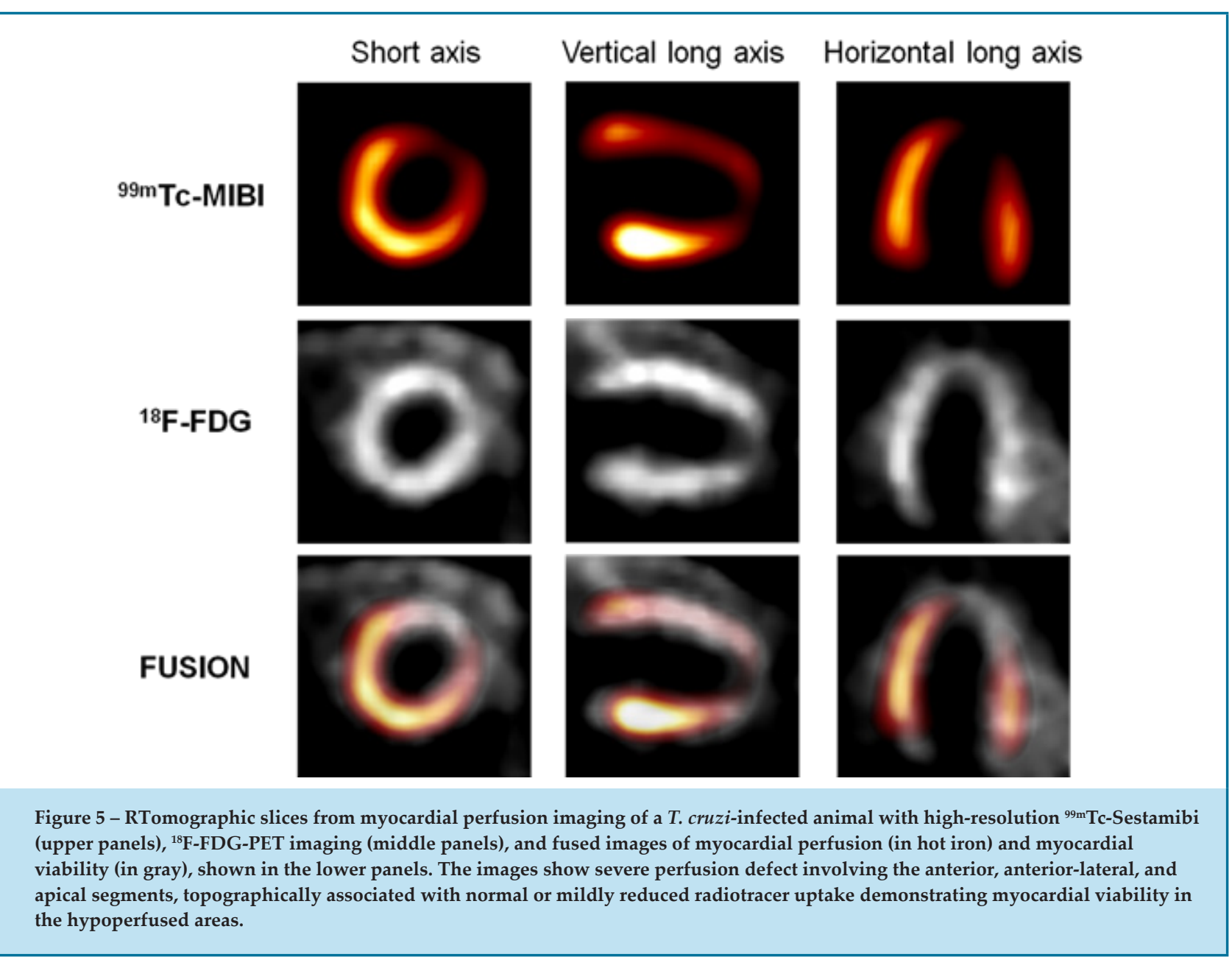


imaging under special metabolic myocardial conditions to suppress cardiomyocyte FDG uptake. Several strategies to suppress cardiomyocyte uptake of ${ }^{18} \mathrm{~F}$-FDG in order to achieve a suitable metabolic state have been reported in clinical and experimental scenarios, including fasting protocols, heparin administration and, in small animals, use of anesthetic combinations such as ketamine and xylazine. Under such conditions, cardiac uptake of FDG is dependent on inflammatory cell infiltrate. ${ }^{42,43}$

However, clinical use of PET imaging for evaluation of inflammation in CCC patients is still limited to a few case reports. The first report was published by Garg et al. ${ }^{44}$ These authors described a CCC patient with dyspnea, acute chest pain, and troponin elevation, but normal coronary angiography. Echocardiography images showed a decrease in LV ejection fraction (35\%) and apical and aneurysmal ballooning of the apex and of the basal inferolateral wall. PET imaging with ammonia $\left({ }^{13} \mathrm{NH}_{3}\right)$ revealed MPD at the apex, basal inferior-lateral, and lateral walls. Additionally, PET imaging with ${ }^{18} \mathrm{~F}-\mathrm{FDG}$ showed diffuse uptake throughout the myocardium. Notably, the most intense focal uptake was adjacent to the apical and basal inferior/ inferolateral aneurysms that had severe ${ }^{13} \mathrm{NH}_{3}$ defects and prominent myocardial delayed enhancement on MRI. ${ }^{44}$ Another clinical case with very similar characteristics was recently reported by Salimy and colleagues. ${ }^{45}$

Shapiro and colleagues reported on two patients with CCC presenting arrhythmic storm with recurrent ventricular tachycardia (VT) and demonstrated higher uptake of ${ }^{18} \mathrm{~F}-\mathrm{FDG}$ in myocardial regions from which the VT originated, suggesting on-going inflammation contributed to triggering VT. ${ }^{46}$ More recently, Moll-Bernardes et al. published a case report of a CCC patient with episodes of sustained ventricular tachycardia and increased uptake of ${ }^{18} \mathrm{~F}$-FDG on PET/CT adjacent to hypoperfused or fibrotic areas. ${ }^{47}$

These initial observations therefore suggest a relationship between myocardial inflammation detected by in vivo FDG-PET imaging and the genesis of severe ventricular arrhythmias in CCC. This is consistent with the structural and functional changes described in myocardial inflammation due to other conditions, i.e., irreversible cell damage with scar formation generating reentrant arrhythmias and exacerbated automaticity within inflamed areas. ${ }^{48}$

Although further studies with larger patient populations are needed, preliminary results suggest that detection of inflammation in vivo using ${ }^{18} \mathrm{~F}$-FDG-PET is a promising tool for monitoring disease progression and even for risk stratification of patients with different degrees of CCC.
Future research should therefore consider these potential new clinical applications of PET imaging.

\section{Conclusions}

CD is a complex illness with multiple pathophysiological mechanisms responsible for its most common and ominous manifestation, CCC. Myocardial changes are distinct at each stage of the disease and radionuclide imaging offers opportunities for detection of diverse cardiac abnormalities throughout the course of disease progression. In the early stages of chronic disease (indeterminate form), and also in patients with the isolated digestive form, it is common to observe impairment of right ventricle function that can be detected by radionuclide ventriculography. In patients with full-blown CCC, coronary microvascular disturbances detectable on myocardial perfusion scintigraphy and cardiac autonomic denervation observed on myocardial scintigraphy with ${ }^{123} \mathrm{I}-\mathrm{mIBG}$ are very prevalent. Thus, an array of nuclear medicine methods constitute useful non-invasive tools for monitoring disease progression and for risk stratification.

Future research should address the efficacy of SPECT and PET images for detecting and monitoring early subclinical myocardial damage in CCC patients as a potential tool for evaluation of therapeutic strategies targeting inflammation and microvascular ischemia.

\section{Acknowledgements}

The authors received grants from the São Paulo Research Foundation - FAPESP (2016/25403-9 and 2017/16450-6).

\section{Potential Conflict of Interest}

No potential conflict of interest relevant to this article was reported.

\section{Sources of Funding}

There were no external funding sources for this study.

\section{Study Association}

This study is not associated with any thesis or dissertation work.

\section{Author Contributions}

Conception and design of the research: Tanaka DM. Critical revision of the manuscript for intellectual content: Marin-Neto JA, Simões MV. 


\section{References}

1. Benziger CP, do Carmo GAL, Ribeiro ALP. Chagas Cardiomyopathy: Clinical Presentation and Management in the Americas. Cardiol Clin. 2017;35(1):31-47.

2. Coura JR, Vinas PA. Chagas disease: a new worldwide challenge. Nature. 2010;465(7301):S6-7.

3. WHO. Chagas disease in Latin America: an epidemiological update based on 2010 estimates. Weekly epidemiological record; 2015; Geneva. Suiça2015. p. 33-4.

4. Moncayo A, Silveira AC. Current epidemiological trends for Chagas disease in Latin America and future challenges in epidemiology, surveillance and health policy. Mem Inst Oswaldo Cruz. 2009;104 Suppl 1:17-30.

5. Prata A. Clinical and epidemiological aspects of Chagas disease. Lancet Infect Dis. 2001;1(2):92-100

6. Marin-Neto JA, Cunha-Neto E, Maciel BC, Simoes MV. Pathogenesis of chronic Chagas heart disease. Circulation. 2007;115(9):1109-23.

7. Oliveira JAMd. Heart Aneurysm in Chagas' Disease. Revista do Instituto de Medicina Tropical de São Paulo. 1998;40(5):301-7.

8. Ribeiro AL, Sabino EC, Marcolino MS, Salemi VM, Ianni BM, Fernandes F, et al. Electrocardiographic abnormalities in Trypanosoma cruzi seropositive and seronegative former blood donors. PLoS Negl Trop Dis. 2013;7(2):e2078.

9. Pazin-Filho A, Romano MM, Almeida-Filho OC, Furuta MS, Viviani LF Schmidt A, et al. Minor segmental wall motion abnormalities detected in patients with Chagas' disease have adverse prognostic implications. Braz J Med Biol Res. 2006;39(4):483-7.

10. Barros MV, da Costa Rocha MO, Ribeiro AL, Machado FS. Tissue Doppler imaging enables the identification of diastolic dysfunction of pseudonormal pattern in Chagas' disease. J Am Soc Echocardiogr. 2001;14(5):353-9.

11. Nunes MC, Dones W, Morillo CA, Encina JJ, Ribeiro AL, Council on Chagas Disease of the Interamerican Society of C. Chagas disease: an overview of clinical and epidemiological aspects. J Am Coll Cardiol. 2013;62(9):767-76.

12. Simões MV, Romano MMD, Marin-Neto JA. Cardiomiopatia da doença de Chagas. In: Magalhães CC, Júnior CVS, Consolin-Colombo FM, Nobre F, Fonseca FAH, Ferreira JFM, editors. Tratado de Cardiologia - SOCESP 3 ed. Barueri: Manole; 2015. p. 705-20.

13. Nunes MCP, Badano LP, Marin-Neto JA, Edvardsen T, FernandezGolfin C, Bucciarelli-Ducci C, et al. Multimodality imaging evaluation of Chagas disease: an expert consensus of Brazilian Cardiovascular Imaging Department (DIC) and the European Association of Cardiovascular Imaging (EACVI). Eur Heart J Cardiovasc Imaging. 2018;19(4):459-60n.

14. Arreaza N, Puigbo JJ, Acquatella H, Casal H, Giordano H, Valecillos $\mathrm{R}$, et al. Radionuclide evaluation of left-ventricular function in chronic Chagas' cardiomyopathy. J Nucl Med. 1983;24(7):563-7.

15. Marin-Neto JA, Bromberg-Marin G, Pazin-Filho A, Simoes MV, Maciel BC. Cardiac autonomic impairment and early myocardial damage involving the right ventricle are independent phenomena in Chagas' disease. Int J Cardiol. 1998;65(3):261-9.

16. Marin-Neto JA, Marzullo P, Sousa AC, Marcassa C, Maciel BC, Iazigi N, et al. Radionuclide angiographic evidence for early predominant right ventricular involvement in patients with Chagas' disease. Can J Cardiol. 1988;4(5):231-6

17. Simoes MV, Oliveira LF, Hiss FC, Figueiredo AB, Pintya AO, Macie $\mathrm{BC}$, et al. Characterization of the apical aneurysm of chronic Chagas' heart disease by scintigraphic image co-registration. Arq Bras Cardiol. 2007;89(2):119-21, 31-4

18. Hagar JM, Rahimtoola SH. Chagas' heart disease. Curr Probl Cardiol. 1995;20(12):825-924.
19. Simoes MV, Pintya AO, Bromberg-Marin G, Sarabanda AV, Antloga CM, Pazin-Filho A, et al. Relation of regional sympathetic denervation and myocardial perfusion disturbance to wall motion impairment in Chagas' cardiomyopathy. Am J Cardiol. 2000;86(9):975-81.

20. Bellotti G, Bocchi EA, de Moraes AV, Higuchi ML, Barbero-Marcial M, Sosa E, et al. In vivo detection of Trypanosoma cruzi antigens in hearts of patients with chronic Chagas' heart disease. Am Heart J. 1996;131(2):301-7.

21. Hagar JM, Rahimtoola SH. Chagas' heart disease in the United States. N Engl J Med. 1991;325(11):763-8.

22. Marin-Neto JA, Simoes MV, Rassi Junior A. Pathogenesis of chronic Chagas cardiomyopathy: the role of coronary microvascular derangements. Rev Soc Bras Med Trop. 2013;46(5):536-41.

23. Torres CM. [Arteriosclerosis of the fine arterial branches of the myocardium (Chagas' coronaritis) \& focal myocytolysis in chronic Chagas' heart disease] Hospital (Rio J). 1958;54(5):597-610

24. Higuchi ML. Endomyocardial biopsy in Chagas' heart disease: pathogenetic contributions. Sao Paulo Medical Journal. 1995;113(2):821-5.

25. Kuschnir E, Kustich F, E[elman M, Santamarina N, Podio RB. Valoration de fluxo miocardico con $\mathrm{Rb} 86$ en pacientes con cardiopatia chagasica con insuficiencia coronaria y en controles normales. Parte 1: Estudios basales. Arq Bras Cardiol. 1974;27:9

26. Marin-Neto JA, Marzullo P, Marcassa C, Gallo Junior L, Maciel BC, Bellina $\mathrm{CR}$, et al. Myocardial perfusion abnormalities in chronic Chagas' disease as detected by thallium-201 scintigraphy. Am J Cardiol. 1992;69(8):780-4.

27. Peix A, Garcia R, Sanchez J, Cabrera LO, Padron K, Vedia O, et al. Myocardia perfusion imaging and cardiac involvement in the indeterminate phase of Chagas disease. Arquivos brasileiros de cardiologia. 2013;100(2):114-7.

28. Hiss FC, Lascala TF, Maciel BC, Marin-Neto JA, Simoes MV. Changes in myocardial perfusion correlate with deterioration of left ventricular systolic function in chronic Chagas' cardiomyopathy. JACC Cardiovasc Imaging. 2009;2(2):164-72.

29. Schwartz RG, Wexler O. Early identification and monitoring progression of Chagas' cardiomyopathy with SPECT myocardial perfusion imaging. JACC Cardiovasc Imaging. 2009;2(2):173-5.

30. Lemos de Oliveira LF, Thackeray JT, Marin Neto JA, Dias Romano MM, Vieira de Carvalho EE, Mejia J, et al. Regional Myocardial Perfusion Disturbance in Experimental Chronic Chagas Cardiomyopathy. J Nucl Med. 2018;59(9):1430-6.

31. Tanaka DM, de Oliveira LFL, Marin-Neto JA, Romano MMD, de Carvalho EEV, de Barros Filho ACL, et al. Prolonged dipyridamole administration reduces myocardial perfusion defects in experimental chronic Chagas cardiomyopathy. J Nucl Cardiol. 2018

32. Koberle F. [Chagas disease: a disease of the peripheral autonomic nervous system]. Wien Klin Wochenschr. 1956;68(17):333-9.

33. Mott KE, Hagstrom JW. The Pathologic Lesions of the Cardiac Autonomic Nervous System in Chronic Chagas' Myocarditis. Circulation. 1965;31:273-86.

34. Köberle F. Pathogenesis of Chagas' disease. Ciba Found Symp. 1974;20:21.

35. Miranda $\mathrm{CH}$, Figueiredo AB, Maciel BC, Marin-Neto JA, Simoes MV. Sustained ventricular tachycardia is associated with regional myocardial sympathetic denervation assessed with 123I-metaiodobenzylguanidine in chronic Chagas cardiomyopathy. Journal of nuclear medicine : official publication, Society of Nuclear Medicine. 2011;52(4):504-10.

36. Landesmann MC, da Fonseca LM, de BPB, do Nascimento EM, Rosado-deCastro PH, de Souza SA, et al. Iodine-123 metaiodobenzylguanidine cardiac imaging as a method to detect early sympathetic neuronal dysfunction in chagasic patients with normal or borderline electrocardiogram and preserved ventricular function. Clinical nuclear medicine. 2011;36(9):757-61.

37. Barizon GC, Simoes MV, Schmidt A, Gadioli LP, Murta Junior LO. Relationship between microvascular changes, autonomic denervation, and myocardial fibrosis in Chagas cardiomyopathy: Evaluation by MRI and SPECT imaging. J Nucl Cardiol. 2018. 
38. Gadioli LP, Miranda CH, Pintya AO, de Figueiredo AB, Schmidt A, Maciel $\mathrm{BC}$, et al. The severity of ventricular arrhythmia correlates with the extent of myocardial sympathetic denervation, but not with myocardial fibrosis extent in chronic Chagas cardiomyopathy : Chagas disease, denervation and arrhythmia. J Nucl Cardiol. 2018;25(1):75-83.

39. Rassi A, Jr., Rassi SG, Rassi A. Sudden death in Chagas' disease. Arq Bras Cardiol. 2001;76(1):75-96.

40. Ferda J, Hromadka M, Baxa J. Imaging of the myocardium using (18) F-FDG-PET/MRI. Eur J Radiol. 2016;85(10):1900-8.

41. Prado CM, Fine EJ, Koba W, Zhao D, Rossi MA, Tanowitz HB, et al. Micro-positron emission tomography in the evaluation of Trypanosoma cruzi-induced heart disease: Comparison with other modalities. Am J Trop Med Hyg. 2009;81(5):900-5.

42. Thackeray JT, Bankstahl JP, Wang Y, Wollert KC, Bengel FM. Clinically relevant strategies for lowering cardiomyocyte glucose uptake for 18F-FDG imaging of myocardial inflammation in mice. Eur J Nucl Med Mol Imaging. 2015;42(5):771-80.
43. Thackeray JT, Bankstahl JP, Wang Y, Korf-Klingebiel M, Walte A, Wittneben A, et al. Targeting post-infarct inflammation by PET imaging: comparison of (68)Ga-citrate and (68)Ga-DOTATATE with (18)F-FDG in a mouse model. Eur J Nucl Med Mol Imaging. 2015;42(2):317-27.

44. Garg G, Cohen S, Neches R, Travin MI. Cardiac (18)F-FDG uptake in chagas disease. J Nucl Cardiol. 2016;23(2):321-5.

45. Salimy MS, Parwani PJ, Mukai K, Pampaloni MH, Flavell RR. Abnormal 18F-FDG and 82Rb PET Findings in Chagas Heart Disease. Clin Nucl Med. 2017;42(5):e265-e8.

46. Shapiro H, Meymandi S, Shivkumar K, Bradfield JS. Cardiac inflammation and ventricular tachycardia in Chagas disease. HeartRhythm Case Rep. 2017;3(8):392-5.

47. Moll-Bernardes RJ, de Oliveira RS, de Brito ASX, de Almeida SA, Rosado-de-Castro PH, de Sousa AS. Can PET/CT be useful in predicting ventricular arrhythmias in Chagas Disease? J Nucl Cardiol. 2020.

48. Muser D, Castro SA, Alavi A, Santangeli P. Potential Role of PET in Assessing Ventricular Arrhythmias. PET Clin. 2019;14(2):281-91. 